\title{
Giant Hepatocarcinoma in Healthy Liver: Case Report
}

\section{Perez-Castilla $\mathrm{A}^{*}$ and Penailillo $\mathbf{P}$}

Department of Digestive Surgery, Clinica Indisa, Chile

*Corresponding author: Perez-Castilla A, Department of Digestive Surgery, Clinica Indisa, University Andres Bello, Santa Maria 1810. Providencia, Santiago, Chile, Tel: 56954111154; Email: investigacionindisa@gmail.com

\section{Case Report}

Volume 2 Issue 3

Received Date: August 01, 2018

Published Date: September 3, 2018

\section{Abstract}

Introduction: Hepatocarcinoma corresponds to the 5th most common cancer worldwide. Multifactorial etiology: infection by HBV or HCV and alcoholic cirrhosis, among the most frequent. Rare in healthy liver. Alpha-1-antitrypsin deficiency within the main causes.

Clinical Case: Male, 65 years old, with no morbid history or alcohol consumption. Consultation for abdominal volume increase. Abdominal MRI confirms giant right hepatic tumor of $17 \mathrm{~cm}$. Serology for HBV and negative HCV, normal alphafetoprotein and normal Ca19-9. Extended right hepatectomy is performed. Definitive biopsy confirms well differentiated hepatocellular carcinoma. Immuno histochemistry identifies intracytoplasmic antitrypsin granules confirming the diagnosis of alpha-1 antitrypsin deficiency.

Discussion: The real incidence of HCC in non-cirrhotic liver is uncertain, ranging between 10 and 50\% of the resected HCC. The deficiency of alpha-1-anti trypsin generates liver disease characterized by the accumulation of the protein in the endoplasmic reticulum of the hepatocyte, which eventually leads to cell damage. This cell damage manifests itself as fibrosis and subsequent cirrhosis, or as a hepatocarcinoma.

Conclusion: Hepatocarcinoma is malignant tumor with known risk factors. It is necessary to consider the deficit of AAT in context of healthy liver.

Keywords: Hepatocarcinoma; Liver transplantation; Giant Tumor

\section{Introduction}

Hepatoma, hepatocellular carcinoma or Hepatocarcinoma (HCC) is the most common malignant primary tumor of the liver ( $>80 \%$ ) [1]. It is defined as a malignant primary epitelial neoplasma of the liver derivad from the hepatocyte. The 5-year survival is currently $12 \%$ and has remained constante despite advances in treatment. It is the fourth cause of cancer mortality. It occurs mainly in men, Rare before the age of 40. It as a poor prognosis if the diagnosis is late [2]. HCC occurs in cirrhotic livers (85\%) associated with risk factors such as chronic infection with hepatitis $C$ virus (HCV) and B (HBV), which increases the risk of HCC up to 20 times compared to uninfected, DHC for alcohol (ALD), NASH, hemocromatosis, Aflatoxins and recent studies 
propase NAFLD, diabetes Mellitus type 2 and Sd. metabolic as precursor causes of HCC [3]. There is a percentage of non-cirrhotic patients who develop the disease, This percentage varies between 10 and 50\% [4]. Some etiologies correspond to viral infection, hemocromatosis, Wilson's disease, alpha-1 antitrypsin (AT) deficiency, among others $[3,5]$.

\section{Case Report}

65 years old man, with no morbid background, no alcohol or drug user. Consultation for volume increase in the upper right quadrant of the abdomen, occasionally manifests colic pain. It is performed as a first approximation CT contrast that reveals large right hepatic tumor. It is completed with abdominal MRI with contrast confirming tumor of $17 \mathrm{~cm}$ that involves the entire right lobe, liver rest without other lesions (Figure 1). Study is complemented with serology for HBV and HCV negative, Alpha-fetus protein 0.6 U / ml, Ca19-9 1U / l.

Extended right hepatectomy was performed (Figure 2) without incidente and with favorable evolution. Definitive biopsy confirms well differentiated hepatocellular carcinoma. Immuno histochemistry identifies intracytoplasmic antitrypsin granules confirming the diagnosis of alpha-1-antitrypsin deficiency.
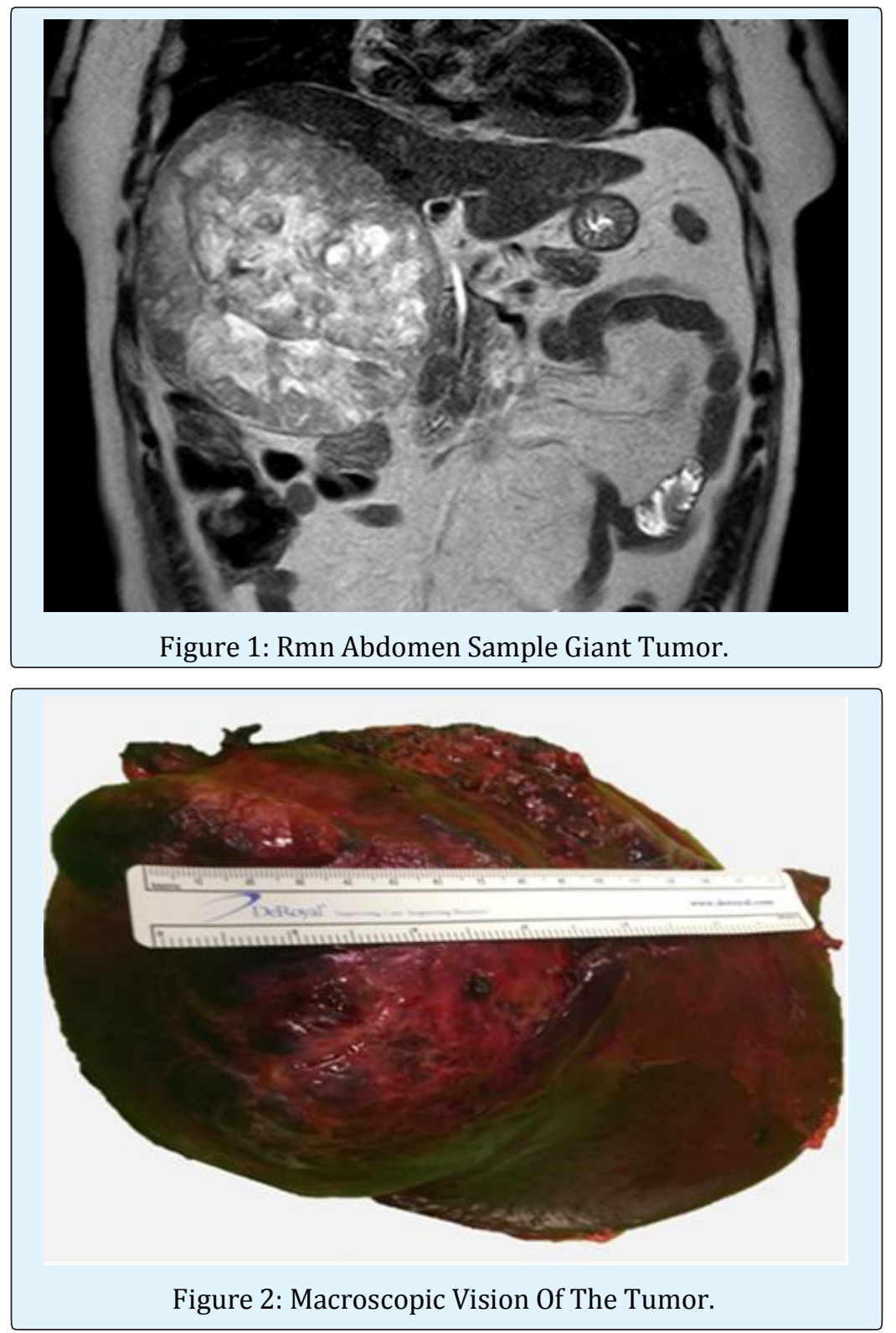


\section{International Journal of Surgery \& Surgical Techniques}

\section{Discussion}

The actual incidence of HCC in non-cirrhotic liver is uncertain, ranging between 10 and $50 \%$ of the resected HCC [4]. Non-cirrhotic patients have absence of the most frequent risk factors. Tumors tend to be of a larger size and stage because of their generally late detection, unlike cirrhotic patients in whom the detection of tumors is earlier due to their more stringent clinical controls $[3,5]$. Therefore, HCC in non-cirrhotic patients can become symptomatic, with abdominal pain being the most frequent antecedent [5]. Regarding treatment, the difference lies in the possibility of performing larger liver resections since the liver remnant is healthy $[3,5]$. The role of liver transplantation as a treatment for these cases is unclear [5].

In relation to life expectancy, it has been found to be greater than in cirrhotic patients, with a 5-year survival between 24 and 54\% [3,5,6]. Prognostic factors have been described as normal alpha-fetoprotein value, single tumor, negative surgical margin, size less than 10 centimeters, absence of vascular invasion [4]. Recurrence in these patients ranges between 27 and $73 \%$, generally within the first 2 years.

The deficiency of $\alpha-1$ AT is defined as the low concentration of alpha-1-anti trypsin in the serum and / or the identification of a defective genotype [7]. It is believed that more than rare is actually underdiagnosed. It is estimated that it affects 1 in 8,000 live births [8]. It is an acute phase protein produced mostly in the liver. Its most important function is the inactivation of proteolytic enzymes in the lung tissue. The disease in the liver is characterized by the accumulation of the protein in the endoplasmic reticulum of the hepatocyte, which eventually leads to cell damage. This cell damage manifests in 2 ways: as fibrosis and subsequent cirrhosis, or as a hepatocarcinoma [7,8]. The malignant transformation of the cell would be determined by a permanent injury condition, directly related to the amount of mutant protein and the number of cells with the alteration. In response, a series of mediators are released to stimulate cell replication, which would act preferentially in healthy cells, until the appearance of the malignant cell follows the adenoma-carcinoma sequence [8].

\section{Conclusion}

Hepatocarcinoma is malignant tumor with known risk factors. Consider the deficit of AAT in context of healthy liver. It is necessary to continue studying this genetic pathology to develop new therapies that are easier to access.

\section{References}

1. Daoudaki M, Fouzas I (2014) Hepatocellular carcinoma. Wiener Medizinische Wochenschrift 164(21-22): 450-455.

2. Parkin DM, Bray F, Ferlay J, Pisani P (2005) Global Cancer Statistics, 2002. CA Cancer J Clin. 55(2):74108.

3. Trevisani F, Frigerio M, Santi V, Grignaschi A, Bernardi M (2010) Hepatocellular carcinoma in noncirrhotic liver: A reppraisal. Digestive and Liver Disease 42(5): 341-347.

4. Bernard PH, Blanc JF, Le Bail B, Ledinghen V, Rullier A, et al. (1998) Carcinome hépatocellulaire sur foie non cirrhotique: un regain d'intérêt. Gastroenterol Clin Biol 22(4): 371-374.

5. Houben KW, McCall JL (1999) Liver transplantation for hepatocelular carcinoma in patients without underlying liver disease: a systematic review. Liver Transpl Surg 5(2): 91-95.

6. Kakar S, Burgart LJ, Batts KP, García J, Jain D, et al. (2005) Clinicopathologic features and survival in fi brolamellar carcinoma: comparison with conventional hepatocellular carcinoma with and without cirrhosis. Mod Pathol 18(11): 1417-1423.

7. Köhnlein T, Welte T (2008) Alpha-1 antitrypsin defi ciency: patogénesis, clinical presentation, diagnosis, and treatment. Am J Med 121(1): 3-9.

8. Rudnick D, Perlmutter D (2005) Alpha-1-antitrypsin defi ciency: A new paradigma for hepatocellular carcinoma in genetic liver disease. Hepatology 42(3): 514-521. 